\title{
Cytonemes extend their reach
}

\section{Thomas B Kornberg}

Cardiovascular Research Institute, University of California, San Francisco, CA, USA. Correspondence to: tkornberg@biochem.ucsf.edu

The EMBO Journal (2013) 32, 1658-1659. doi:10.1038/emboj.2013.115; Published online 14 May 2013

Cytonemes are specialized filopodia, first described in the Drosophila wing imaginal disc, that were proposed to mediate long distance signalling during development. A recent report from Barna and colleagues (Sanders et al, 2013) published in Nature shows that cytonemes in the chick limb bud carry SHH signalling proteins out to signal receiving cells, thus highlighting their evolutionarily conserved roles in cell-cell communication.

Our textbooks inform us that long distance signalling by animal cells is carried out in either of two ways. One, specific for neurons, involves signal exchange at sites of direct contact. The cell body of a neuron can be far from target cells (even metres away), but neurons extend processes that can bridge the distance to the target cell. Non-neuronal cells, in contrast, are hemmed in by their neighbours, their contacts limited to nearest neighbours, and they use the other mode: signal release at the surface of signal-producing cells, followed by dispersion in extracellular fluid so that released signals eventually bind to receptors on target cells.

This clean dichotomy of form and function that has long been thought to distinguish neurons from all other cells appears no longer valid. Several lines of evidence now show that non-neuronal cells also extend asymmetric processes-filopodia-that mediate long-range signalling. Filopodia are rod-like projections that extend from the periphery of many type of cells (for review, see Faix et al, 2009). They contain bundles of actin filaments, are highly dynamic and flexible, and can appear to surf over the surface of cells. They are present at nerve growth cones, where they project towards sources of chemotactic signals and are presumed to have a sensory, pathfinding function. Some tumour cells have such processes (a.k.a. invadopodia), which may be involved in ECM degradation and intravasation during metastasis. They are present in cells at the leading edge of epithelial sheets, in fibroblasts, myoblasts, glia, and dendritic cells. Although the functions of these filopodia have not been confirmed-loss-of-function studies are complicated by general effects on the cytoskeleton-the settings in which they are found suggest roles in migration, adhesion, and cell-cell recognition. Nevertheless, their presence in so many cell types informs us that neurons are not unique in making projections that reach into the surrounding milieu.

Cytonemes are filopodia that are specialized for transmitting signalling proteins between communicating cells. The idea is based on a model in which filopodia are structurally and functionally diverse, with a subset dedicated and designed for juxtacrine signalling between distant cells. The term cytoneme was coined to highlight this specialized functionality (Ramirez-Weber and Kornberg, 1999). Filopodia with apparent sensory function had been observed previously in various contexts: thin, dynamic filopodia extend across sea urchin embryos, appearing to explore and perhaps pattern distant sheets of cells (reviewed in McClay, 1999), and anthropomorphic descriptions of neuronal growth cone

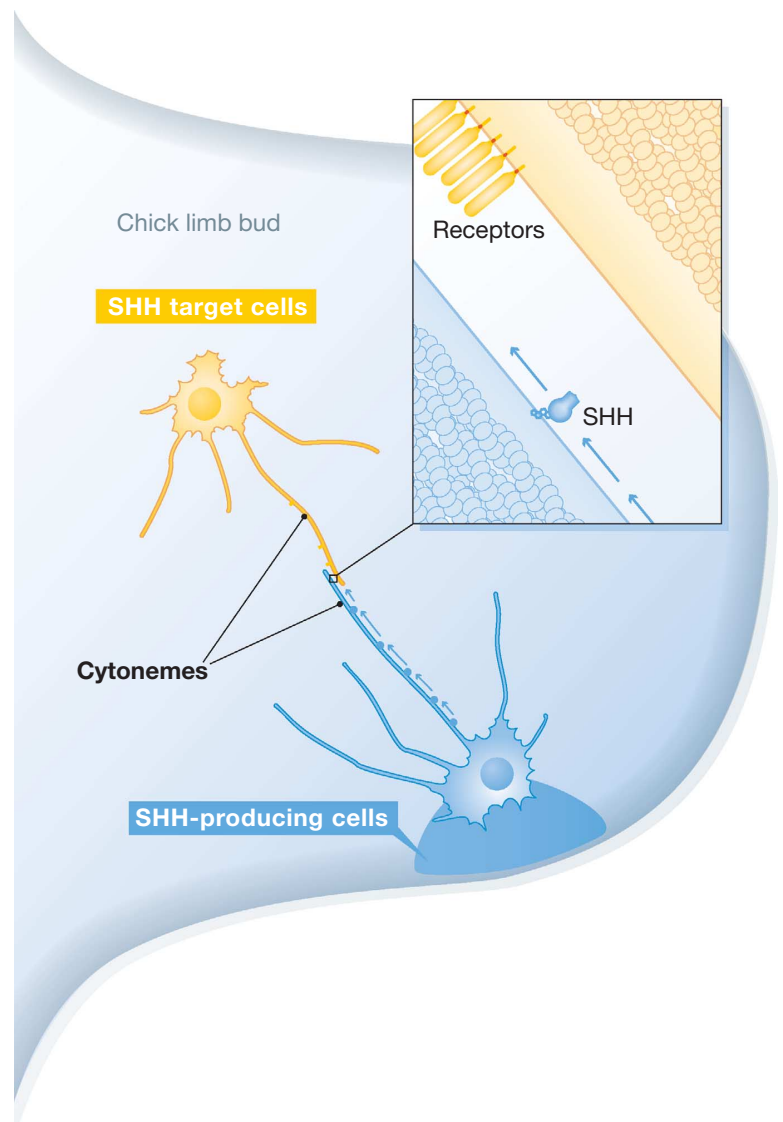

Figure 1 Cytonemes in the Hedgehog domain. Drawing depicting a spatial concentration gradient of SHH extending across the limb bud, and showing cells that span this gradient with cytonemes that either carry SHH from the distal edge (blue) or reach out with cytonemes that carry SHH receptors (yellow). Contacts between these cytonemes may mediate transport of SHH to target cells. 
filopodia imply sensory roles. The discovery of filopodia associated with known signalling systems in Drosophila imaginal discs ties this filopodia type directly to cell-cell signalling (Hsiung et al, 2005; Roy et al, 2011).

The recent report from the Barna lab adds beautiful new data supporting the concept of contact-mediated long distance signalling by non-neuronal cells (Sanders et al, 2013). In the interest of full disclosure, I helped mentor Maria during much of her 5-year tenure as a Sandler Fellow at UCSF, and fondly recall that day she emerged from her microscope room with the news, 'Cytonemes!'. Our early work identified cytoneme-like filopodia in explants of chick embryos (Ramirez-Weber and Kornberg, 1999), but this new study, which applied real-time imaging to living embryos and monitored long-range transport of sonic hedgehog ( $\mathrm{SHH}$ ) proteins in the developing chick limb bud, adds strong evidence for cytoneme presence and function.

There have been several previous reports of cytoneme-associated Hh in Drosophila. Studies of isolated wing imaginal discs (Callejo et al, 2011; Bilioni et al, 2013) showed that Hhproducing cells package $\mathrm{Hh}$ in puncta that distribute along cytonemes, and that Hh-containing cytonemes extend from both Hh-sending and -receiving cells. The Hh co-receptors iHog and Boi were also shown to co-localize in cytonemes. And in the female germline stem cell niche, Hh localizes in short cytonemes in the producing cells (Rojas-Rios et al, 2012).

The new paper from Sanders et al (2013) adds impressive technical virtuosity and ingenuity to extend these findings to a vertebrate embryo. These investigators developed a transposon-mediated expression system and a

\section{References}

Bilioni A, Sanchez-Hernandez D, Callejo A, Gradilla AC, Ibanez C, Mollica E, Carmen Rodriguez-Navas M, Simon E, Guerrero I (2013) Balancing hedgehog, a retention and release equilibrium given by Dally, Ihog, Boi and shifted/dWif. Dev Biol 376: 198-212

Callejo A, Bilioni A, Mollica E, Gorfinkiel N, Andres G, Ibanez C, Torroja C, Doglio L, Sierra J, Guerrero I (2011) Dispatched mediates Hedgehog basolateral release to form the long-range morphogenetic gradient in the Drosophila wing disk epithelium. Proc Natl Acad Sci USA 108: 12591-12598

Faix J, Breitsprecher D, Stradal TE, Rottner K (2009) Filopodia: complex models for simple rods. Int J Biochem Cell Biol 41: 1656-1664

Hsiung F, Ramirez-Weber FA, Iwaki DD, Kornberg TB (2005) Dependence of Drosophila wing imaginal disc cytonemes on Decapentaplegic. Nature 437: 560-563 custom-designed confocal microscope to image fluorescent proteins in chick embryos with unprecedented sensitivity and resolution. Focusing on the limb bud where $\mathrm{SHH}$ is expressed by a small group of mesenchymal cells at the posterior margin, they show that a non-cholesterol modified form of $\mathrm{SHH}$ is carried out from SHH-producing cells by actinbased filopodia in $200 \mathrm{~nm}$ particles that move at rates consistent with actin-based myosin motors, that the $\mathrm{SHH}$ co-receptors $\mathrm{CDO}$ and BOC are present in filopodia that extend from SHH-receiving cells, and that the filopodia that extend from SHH-producing and -responding cells are highly dynamic and can be as long as $150 \mu \mathrm{m}$, together encompassing the entire $300 \mu \mathrm{m}$ length of the $\mathrm{SHH}$ signalling field (see Figure 1).

Although this work characterizes a form of SHH that is not modified with cholesterol and although the work does not show that limb bud cytonemes deliver SHH for uptake by receiving cells, it puts to rest the idea that cytonemes are limited to the surface of epithelia, incapable of navigating through a three-dimensional tissue. Therefore, the question for future research becomes not whether, but how these organelles navigate in and around organs. However, by showing that flies and vertebrates handle $\mathrm{Hh}$ in similar ways, this work lends credence to the idea that long distance signalling shares fundamental similarities in all animals and in all tissues and cell types.

\section{Conflict of interest}

The author declares that he has no conflict of interest.
McClay DR (1999) The role of thin filopodia in motility and morphogenesis. Exp Cell Res 253: 296-301

Ramirez-Weber FA, Kornberg TB (1999) Cytonemes: cellular processes that project to the principal signaling center in Drosophila imaginal discs. Cell 97: 599-607

Rojas-Rios P, Guerrero I, Gonzalez-Reyes A (2012) Cytonememediated delivery of hedgehog regulates the expression of bone morphogenetic proteins to maintain germline stem cells in Drosophila. PLoS Biol 10: e1001298

Roy S, Hsiung F, Kornberg TB (2011) Specificity of Drosophila cytonemes for distinct signaling pathways. Science 332: 354-358

Sanders TA, Llagostera E, Barna M (2013) Specialized filopodia direct long-range transport of $\mathrm{SHH}$ during vertebrate tissue patterning. Nature (advance online publication 28 April 2013; doi:10.1038/nature12157) 